\title{
Differential Response of Rice Hybrids and Varieties to Nitrogen Fertilization and their Exploitation in Andaman and Nicobar Islands
}

\author{
B. Gangaiah*, Adamala Sirisha, S. Swain and T. Subramani \\ Division of Natural Resource Management, ICAR-Central Island Agricultural Research \\ Institute, Port Blair, Andaman \& Nicobar Islands, India \\ *Corresponding author
}

\section{A B S T R A C T}

\section{Keywords}

Rice, Hybrid, Nitrogen, Net income, High yielding variety

Article Info

Accepted:

20 August 2019

Available Online:

10 September 2019
In Andaman and Nicobar Islands, India, rice productivity is low due to traditional long duration variety (C-14-8) cultivation on poorly fertile soils with minimal or no fertilizer use due to heavy rains. In this context, ability of rice hybrids and high yielding varieties (HYV) under varying nitrogen supplies to adopt and enhance rice productivity and profits. Field study was made during 2015 rainy season in split plot design with three replications. Treatments formed by combination of 5 rice cultivars (3 hybrids: KRH-4, 28P09 \& DRRH-3 and two HYV: WGL-14 and CARI Dhan-6) in main plot and four nitrogen (N) rates $(0,50,100$ and $150 \mathrm{~kg} / \mathrm{ha})$ as sub-plot treatments. Best performing cultivars $(\mathrm{KRH}-4$ hybrid and WGL-14 variety) and nitrogen rate (100 kg) were evaluated in 2016 and 2018 seasons. Results (2015 season) have indicated that KRH-4 and 28P09 hybrids have 39.2 and $28.2 \%$ yield improvements over local HYV CARI Dhan-6 (2.09 t/ha). Above higher yields of hybrids with similar cost of cultivation as that of a variety have brought 3.16-4.52 times more profits. Grain yield exhibited linear response to nitrogen up to $150 \mathrm{~kg}$ rate, however, its application beyond $100 \mathrm{~kg}$ was not economically rewarding. Rice cultivar and nitrogen interaction indicated that KRH-4 hybrid was suitable for both no, low and high $\mathrm{N}$ inputs. Rice hybrid '28P09' required N fertilization $(100 \mathrm{~kg})$ for its potential performance. Confirmatory trails of KRH-4 for two seasons (2016 \& 2018) have shown $17.1 \%$ yield advantage over HYV 'WGL-14' over 3 seasons at $100 \mathrm{~kg} \mathrm{~N}$ rate. The study proved that hybrids are better candidates for Island ecosystem where response to $\mathrm{N}$ fertilization is rainfall dependent and soils have inherent fertility.

\section{Introduction}

Andaman and Nicobar Islands (ANI), an Union Territory of India lies as a separate land mass $\left(0.8249 \mathrm{~m} \mathrm{~km}^{2}\right)$ from mainland in the midst of Bay of Bengal at a distance of over $1100 \mathrm{~km}$. ANI is inhabited by $0.38 \mathrm{~m}$ people as per 2011 census and is frequented by 0.487 $\mathrm{m}$ tourists during 2017. Farming is practised on 40506 ha of which rice (Oryza sativa L.) crop accounts for $13.2 \%$ acreage (DOES, 2018). Rice is the only cereal staple grown in ANI as a transplanted crop of rain fed lowlands near the coast on acidic soils with salinity dimension (acid-saline, acid-sulphatesaline) with high phosphorus fixation and low available nitrogen and potassium (Singh et al., 1988). Excess monsoon rains ( $200 \mathrm{~cm}$ in $\sim 90$ days) of islands results in flash flooding; deep water submergence of paddy soils (Courtois $e t$ 
al., 2001) and have poor crop yields (Amanullah et al., 2007). Untimely rains during rice season often hamper timely nitrogen fertilizer top dressing. Even if applied timely, its efficacy is reduced with subsequent rains through various losses especially run off. Nutrient omission studies of NPK have indicated a yield penalty of $60.7 \%$ in the study region (Gangaiah et al., 2016) that would be still higher in no soil amelioration (amendments) scenario of islands. In such edapho-climatic condition of ANI, long duration, tall, photosensitive varieties (C-14-8) requiring minimal or no fertilizer nitrogen inputs have been cultivated on as high as $70 \%$ total acreage (Subramani et al., 2014) with low yields and thus profits. Poor economic prospects of rice crop has resulted in loss of patronage with its cultivation as evident from the decreased area from 12000 ha in late 1990s (Mandal et al., 2004) to the current 5340 ha (DOES, 2018). Indian Ocean Tsunami of $26^{\text {th }}$ December, 2004 has further reduced the rice crop prospects due to deteriorated soil and water quality (Ganesh Kumar et al., 2009) that however, were restored to normalcy owing to leaching /washing of the soils of salts by copious rainfall of the islands (Velmurugan et al., 2015) and have overcome the constrained yields and profits as in states of Tamil Nadu and Pondicherry (Shanmugasundaram and Ponnusamy, 2009) that have low rainfall. Attempts are made to improve the yield potential of local rice varieties through breeding (Singh et al., 2014) and also introduction of high yielding varieties from mainland to some extent. Starting from 1995 with release of first hybrid (APRRH-1), India has released 97 hybrids till 2017 (DRD, 2018) with 15-20\% yield gains (FAO, 2014). Hybrids with higher and more vigorous root systems (Yang and Sun 1986; Zhang et al., 2009) than a variety were found to contribute to more soil $\mathrm{N}$ tapping (Hunag et al., 2017) makes them ideal candidates for low $\mathrm{N}$ input farming of islands. It is in this context, hybrids were introduced and tested in Islands and is aimed at understanding the rice cultivar and $\mathrm{N}$ interactions and tapping the same for islands where $100 \mathrm{~kg} / \mathrm{ha} \mathrm{N}$ (Damodaran et al., 2012) is recommended dose for a rice variety.

\section{Materials and Methods}

\section{Experimental location}

Field studies on rain fed lowland transplanted rice was conducted during July- November, 2015 -2018 at the Bloomsdale farm, ICARCentral Island Agricultural Research Institute, Andaman, \& Nicobar Islands, India located at $11^{0} 38^{\prime} 06^{\prime \prime} \mathrm{N}$ latitude and $92^{\circ} 39^{\prime} 15^{\prime \prime} \mathrm{E}$ longitude at an altitude of $14 \mathrm{~m}$-above mean sea level. This study region has Udic moisture and Isothermic temperature regime.

\section{Experimental soil characteristics}

The experimental clay loam soil, at start of study in $0-20 \mathrm{~cm}$ depth has $6.3 \mathrm{pH}$ (measured in a 1:2.5 soil-water suspension), non-saline $(\mathrm{ECe}<0.58 \mathrm{dS} / \mathrm{m}), 6200 \mathrm{~kg} / \mathrm{ha}$ organic carbon (Walkley and Black method, Jackson, 1973), $258 \mathrm{~kg} / \mathrm{ha}$ alkaline permanganate hydrolysable $\mathrm{N}$ (Subbiah and Asija,1956), $11.0 \mathrm{~kg} / \mathrm{ha}$ 0.5M $\mathrm{NaHCO}_{3}$ extractable $\mathrm{P}$ (Olsen's method, Olsen et al., 1954) and $132 \mathrm{~kg} / \mathrm{ha} 1 \mathrm{~N} \mathrm{NH_{4 } \mathrm { OAC }}$ exchangeable $\mathrm{K}$ (Flame photometer method, Jackson, 1973) as determined by using the procedures described by Singh et al., (2005).

\section{Treatments}

Treatments during 2015 study consisted of five recently improved rice cultivars (28P09, DRRH-3, KRH-4 rice hybrids; WGL-14 a high yielding varieties from mainland and CARI Dhan-6 a local improved variety) as main plot and four nitrogen rates $(0,50,100$ and $150 \mathrm{~kg} / \mathrm{ha}$ ) in sub-plot. These nitrogen rates represented $0,50,100$ and $150 \%$ of 
recommended dose of rice crop in experimental region. Treatments were replicated thrice in a split plot design. Best performing hybrid (KRH-4) from 2015 study was re-evaluated against the variety 'WGL14 ' at $4 \mathrm{~N}$ levels (2016) and during 2017: 28P09 and CARI Dhan-6 and during 2018: KRH-4 and WGL-14 at recommended dose of $\mathrm{N}$ i.e. $100 \mathrm{~kg} / \mathrm{ha}$ were evaluated as a part new hybrids screening trials. Package of practices remained the same.

\section{Crop nutrition}

Nitrogen as prilled urea $(46.4 \% \mathrm{~N})$ was applied in three equal splits on $5^{\text {th }}, 27^{\text {th }}$ and $47^{\text {th }}$ day after transplanting (DAT) rice crop. A sub-plot size of $5 \mathrm{~m} \times 3 \mathrm{~m}$ with a $1 \mathrm{~m}$ channel between plots on all sides was formed by 30 $\mathrm{cm}$ high soil levee to contain inter plot $\mathrm{N}$ movement. Main plots and replications were separated by $2 \mathrm{~m}$ alleys of bunds and a channel. Irrigation water was also applied to each plot separately. Thus, inter-plot movement of $\mathrm{N}$ was fully controlled. The experimental area received uniformly $60 \mathrm{~kg} / \mathrm{ha}$ each of $\mathrm{P}_{2} \mathrm{O}_{5}$ and $\mathrm{K}_{2} \mathrm{O}$ as single super phosphate $\left(16 \% \mathrm{P}_{2} \mathrm{O}_{5}\right)$ and potassium chloride $\left(60 \% \mathrm{~K}_{2} \mathrm{O}\right)$ applied in last puddling prior to levelling and field layout. Land preparation

The experimental soil was thoroughly prepared by three times power tiller puddling after saturating the soil with water for a week. First puddling was done with no standing water that inverted and incorporated the existing natural grasses and other plants. In second and third puddling, $2-5 \mathrm{~cm}$ standing water was maintained for pulverising the soil. Soil was allowed to settle for a day, was manually levelled and experimental lay out was done.

\section{Nursery and main field crop management}

Clean paddy seeds were soaked in water for 24 hours in a bucket followed by placing them in gunny bag for 24 hours for promoting germination with frequent watering. In a thoroughly prepared nursery field, five raised beds of $1 \mathrm{~m}$ width, $5 \mathrm{~m}$ length and $0.20 \mathrm{~m}$ height were made with drainage channels on all sides. Seeds $(0.5 \mathrm{~kg})$ of each variety were sown on separate bed on $1^{\text {st }}$ July, 2015. Nitrogen (urea) was applied @ $2 \mathrm{~g} \mathrm{~N} / \mathrm{m}^{2}$ on 5 and $20^{\text {th }}$ day. Nursery was watered daily and weeds were removed once on $25^{\text {th }}$ day after seeding. Thirty (30) day old seedlings were uprooted and transplanted in main field on $30^{\text {th }}$ July, 2015 using 2 seedlings/ hill located at $20 \times 15 \mathrm{~cm}$ spacing. During 2016, Rice crop was grown under rain fed conditions and faced no moisture stress during its life cycle as need based irrigations were given. Manual weeding was done twice at 25 and 45 days after transplanting (DAT) prior to $2^{\text {nd }}$ and $3^{\text {rd }} \mathrm{N}$ topdressing.

\section{Data recording}

Days to $50 \%$ flowering (visible observation) and physiological maturity were recorded (from seed soaking to flowering / maturity) and reported. Plant height $(\mathrm{cm})$ of 10 randomly selected hills from ground to tip of the top most leaf (flag leaf) was measured and the panicles were counted prior to harvest treatment wise. The same hills were harvested $5 \mathrm{~cm}$ above the ground level and weight $(\mathrm{g})$ was recorded. Grains were separated by hand from the above 10 hills and counted manually. Average number of grains/panicle was estimated by dividing number of grains with panicle number. Grain and straw was oven dried to bring down the moisture contents to 14 and 10\% respectively and weight was recorded. Harvest index was estimated as ratio of weight of 10 hills grain (14\% moisture) to weight of straw (10 moisture) + grain. Weight of 1000 oven dried grains was recorded and reported as test weight (g). Crop was harvested plot wise and biomass yield $(\mathrm{kg})$ was recorded. It was allowed to dry in the shade of threshing floor for two days and 
threshed by manually operated pedal thresher. Grain yield was recorded plot wise. Grain and straw yields were adjusted to 14 and $10 \%$ moisture level and their total weight is taken as biological yield /plot. From plot yields, per ha yields were estimated.

\section{Nitrogen uptake and use efficiency}

Nitrogen $(\mathrm{N})$ concentration of grain and straw was estimated as per procedures Singh et al., (2005) and uptake was estimated as product of grain/ straw yield (t/ha) x nutrient concentration (\%) / 100. Nitrogen use efficiencies were calculated as per Fageria and Baligar (2011).

AE: Agronomic efficiency (kg grain/ $\mathrm{kg} \mathrm{N}$ applied): Grain yield in $\mathrm{N}$ applied plot (kg/ha) - grain yield in no $\mathrm{N}$ applied plot/ $\mathrm{N}$ fertilizer applied (kg/ha)

PE: Physiological efficiency (kg biomass/ kg $\mathrm{N}$ uptake): Biomass (grain + straw) yield in $\mathrm{N}$ applied plot $(\mathrm{kg} / \mathrm{ha})$ - Biomass yield in no $\mathrm{N}$ applied plot / $\mathrm{N}$ uptake in $\mathrm{N}$ applied - No $\mathrm{N}$ applied plot

AR: Apparent recovery (\%): ( $\mathrm{N}$ uptake by biomass in fertilized-N uptake by biomass in unfertilized plot/ nitrogen applied) x 100

UE: Utilization Efficiency (kg/kg): Physiological efficiency x Apparent recovery NHI: Nitrogen harvest index: (Grain uptake / biomass uptake) x 100

\section{Economics}

Economics for 2015 study were estimated based input prices of market and output price of rice grain as announced by Government of India as minimum support price (Rs. 14,100/tonne, 2015-16) and assumed straw price of Rs. 2,000/tonne. Benefit Cost ratio as worked out as ratio of gross income grain yield (t/ha) x 14100 +straw yield (t/ha) $\mathrm{x}$ 2000 \} to cost of cultivation (Rs/ha). A fertilizer price of $12.87 / \mathrm{kg} \mathrm{N}$ was used.

\section{Statistical analysis}

The analysis of variance was done in Split Plot Design and significance of treatment differences was compared by critical difference at $5 \%$ level of significance $(\mathrm{P}=0.05)$ and statistical interpretation of treatments was done as per Gomez and Gomez (1984).

\section{Results and Discussion}

\section{Weather during study period}

Weather data during the experiment period was highly congenial for rice cultivation (Figure 1). A rain fall of $140.2 \mathrm{~cm}$ was received in 62 rainy days. A mean maximum and minimum temperature of 30.2 and $24.7^{\circ} \mathrm{C}$ and relative humidity of $75-90 \%$ was recorded at the Indian Meteorological Department (IMD)weather station at Port Blair during crop life cycle (July-November). Crop required irrigations during October month to maintain $3-5 \mathrm{~cm}$ standing water and were given through ground water. Required plant protection measures were given to go crop free of pest induced losses. Weeds were taken take through manual weeding. Recommended P, K fertilizers were applied uniformly to exclude their yield limitations in rice crop. There was uniform bird damage to grains at experimental site that could not be controlled as the birds are under Forest Protection (wild life) laws. It is a common factor for all treatments like $\mathrm{P}$ and $\mathrm{K}$ fertilizers. Thus all biotic and abiotc stresses of rice crop were taken care and any differences in crop performance was solely ascribed to cultivars (hybrid/ variety), nitrogen fertilization and their interaction. The results of study were presented in order of cultivar, nitrogen and their interaction effects. 


\section{Rice cultivars}

\section{Plant height and yield attributes}

Rice cultivars differed greatly for plant height and yield attributes (Table 1). 'KRH-4' hybrid being at a par with '28P09' hybrid has produced significantly taller plants than other cultivars. CARI Dhan- 6 has produced the shortest plants. Cultivars differed by 2-6 days for reaching to $50 \%$ flowering and physiological maturity stage, however, these differences were statistically insignificant. Significantly higher number of panicles $/ \mathrm{m}^{2}$ (317.5), grains/panicle (123.8) and 1000 grain weight $(25.07 \mathrm{~g})$ was recorded by KRH-4, 28P09 and CARI Dhan-6, respectively. Panicles $/ \mathrm{m}^{2}$ of 'KRH-4' \& 'WGL-14 and '28P09' \& 'DRRH-3' were at par. CARI dhan- 6 has produced the least panicles $/ \mathrm{m}^{2}$. Based on 1000 grain weight, WGL-14 (16.63 g) has lowest values, 28P09 and KRH-4 (19.33 and $19.90 \mathrm{~g}$ ) and DRRH-3 and CARI dhan-6 (23.9 and $25.07 \mathrm{~g})$ formed two separate groups. Significantly higher number of grains/ panicle was recorded by KRH-4 than other four cultivars which in turn have at par values among themselves.

\section{Grain and biomass yield}

Rice hybrid 'KRH-4' has out yielded all other cultivars significantly for grain and biomass (2.91 and 9.20 t/ha) yields (Table 2) and 'CARI Dhan-6' stood at the bottom with significantly lower values (2.09 and $6.64 \mathrm{t} / \mathrm{ha}$ ). Hybrid '28P09' is the second best performer for grain and biomass yields. DRRH-3 hybrid and 'WGL-14' variety have at par yields. Cultivars did not differ for harvest index and have a mean value of 32.18 .

\section{Nitrogen uptake and use efficiency}

Rice cultivars differed for grain and biomass (grain + straw) $\mathrm{N}$ uptake (Table 3). 'KHR-4' hybrid has significantly higher $\mathrm{N}$ uptake values (grain and biomass) than all other cultivars; however, its grain $\mathrm{N}$ uptake was at par with '28P09' hybrid. 'DRRH-3' hybrid and 'WGL-14' variety have at par $\mathrm{N}$ uptake values while CARI Dhan-6 has the least uptake values (grain and biomass) and its grain $\mathrm{N}$ uptake was at par with WGL-14.

Nitrogen harvest index (NHI) remained unaffected by cultivars. For $\mathrm{N}$ use efficiency indices, 28P09 and CARI Dhan - 6 have significantly higher and lower values, respectively. However PE values are least in 'WGL-14' variety.

\section{Economics}

Economics (Rs/ha) of rice cultivation (Table 4) revealed that hybrids have Rs. 3600/ha higher cost of cultivation than a variety (Rs. 34851) on account of high seed price (Rs. 200 and 30 per $\mathrm{kg}$ of hybrid and variety) while seed rate used remained same for both. Net income (Rs/ha) of 'KRH-4' was significantly higher (Rs.16616) than all other cultivars. Other cultivars in descending order of net income are: 28P09> WGL-14>DRRH3>CARI Dhan-6. Benefit cost ratio (BCR) followed the net income and was the highest in 'KRH-4' (1.38) and CARI Dhan-6 has the least BCR (1.10) which in turn was at par with DRRH-3 (1.12).

\section{Nitrogen fertilization impacts}

\section{Plant height and yield attributes}

Plant height and yield attributes (except 1000 grain weight) of rice varied greatly due to $\mathrm{N}$ fertilization (Table 5). Successive increase of $50 \mathrm{~kg} \mathrm{~N}$ fertilization from 0 to 100 for plant height and panicles $/ \mathrm{m}^{2}$, and from 0 to $50 \mathrm{~kg}$ in case of grains / panicle have brought marked improvements in their values over immediate preceding $\mathrm{N}$ rate. 
Fig.1 Weather data of study site (rainfall, rainy days on $\mathrm{y}_{1} \&$ temperature on $\mathrm{y}_{2}$ axis).

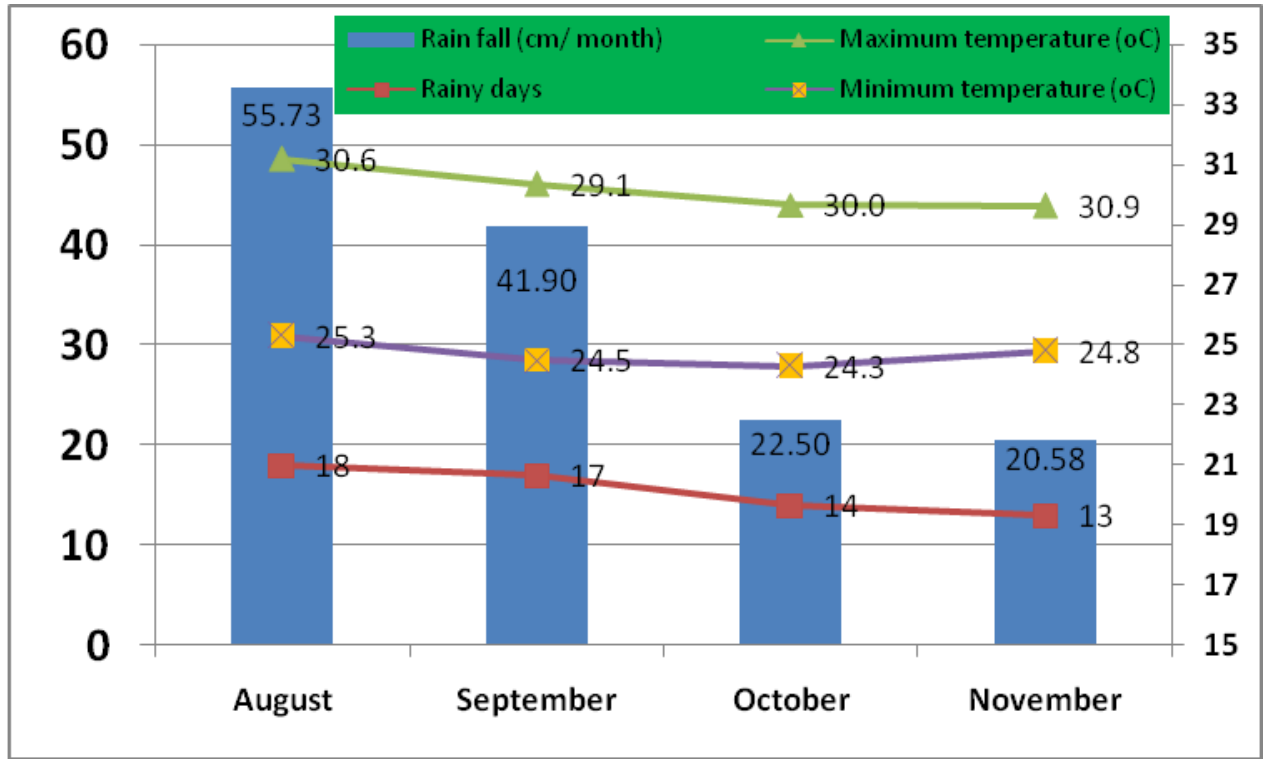

Fig.2 Nitrogen concentration of rice grain and under varying nitrogen rates

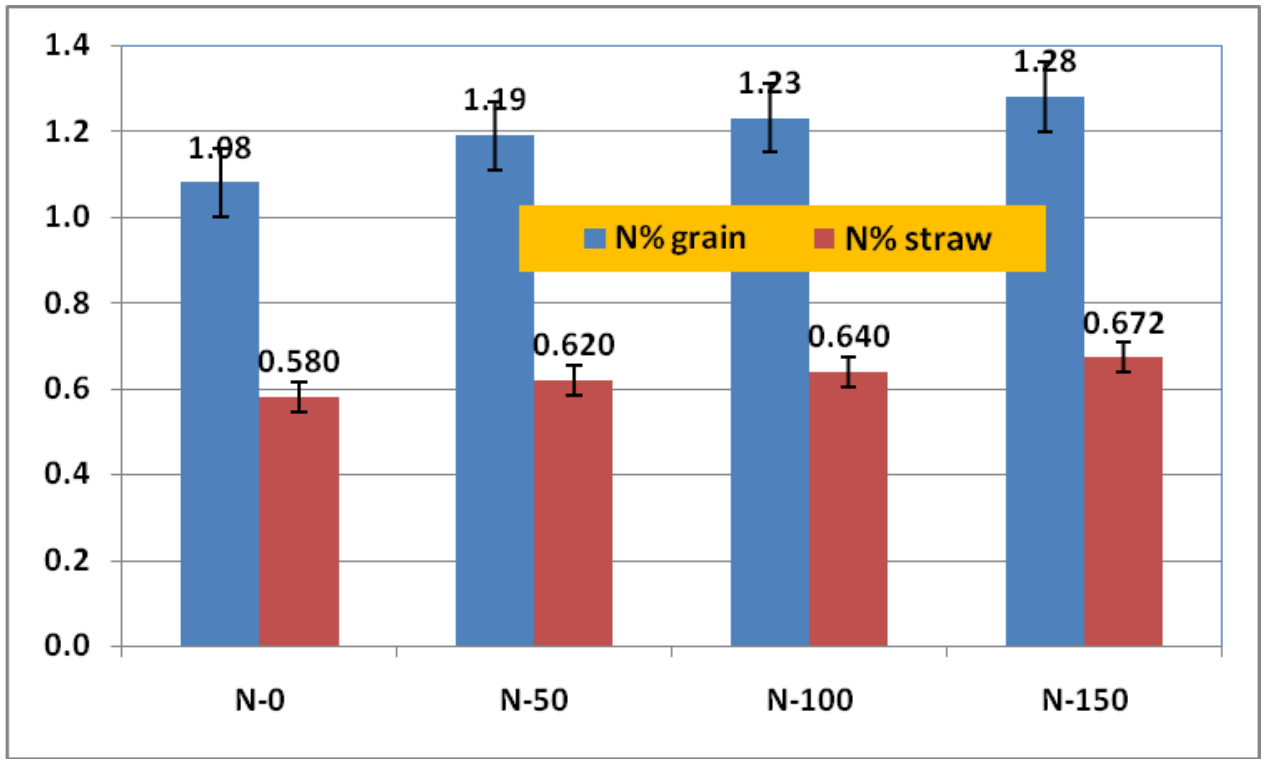


Table.1 Growth and yield attributes of rice as affected by its cultivars

\begin{tabular}{|l|c|c|c|c|c|c|}
\hline $\begin{array}{l}\text { Rice hybrid / } \\
\text { variety* }\end{array}$ & $\begin{array}{c}\text { Plant height } \\
\text { (cm) at harvest }\end{array}$ & $\begin{array}{c}\text { Days to 50\% } \\
\text { flowering }\end{array}$ & $\begin{array}{c}\text { Days to } \\
\text { maturity }\end{array}$ & $\begin{array}{c}\text { Panicles/ } \\
\mathbf{m}^{\mathbf{2}}\end{array}$ & $\begin{array}{c}\text { Grains } \\
\text { /panicle }\end{array}$ & $\begin{array}{c}\text { Test } \\
\text { weight } \\
\text { (g) }\end{array}$ \\
\hline $\mathbf{2 8 P 0 9}$ & $123.6^{\mathrm{c}}$ & $103^{\mathrm{a}}$ & $129^{\mathrm{a}}$ & $274.0^{\mathrm{b}}$ & $123.8^{\mathrm{c}}$ & $19.33^{\mathrm{b}}$ \\
\hline DRRH-3 & $110.8^{\mathrm{b}}$ & $101^{\mathrm{a}}$ & $125^{\mathrm{a}}$ & $258.6^{\mathrm{b}}$ & $100.4^{\mathrm{a}}$ & $23.90^{\mathrm{c}}$ \\
\hline KRH-4 & $125.0^{\mathrm{c}}$ & $100^{\mathrm{a}}$ & $123^{\mathrm{a}}$ & $317.5^{\mathrm{c}}$ & $105.0^{\mathrm{ab}}$ & $19.91^{\mathrm{b}}$ \\
\hline WGL-14* $^{\mathrm{b}}$ & $112.7^{\mathrm{b}}$ & $100^{\mathrm{a}}$ & $126^{\mathrm{a}}$ & $303.7^{\mathrm{c}}$ & $108.7^{\mathrm{b}}$ & $16.62^{\mathrm{a}}$ \\
\hline CARI Dhan-6* & $105.3^{\mathrm{a}}$ & $98^{\mathrm{a}}$ & $123^{\mathrm{a}}$ & $223.2^{\mathrm{a}}$ & $107.0^{\mathrm{b}}$ & $25.07^{\mathrm{c}}$ \\
\hline CD (P=0.05) & 5.15 & NS & NS & 17.02 & 5.11 & 2.101 \\
\hline
\end{tabular}

Note: same superscript alphabets represent statistically similar values

Table.2 Grain, biomass yield and harvest index of rice - cultivars

\begin{tabular}{|l|l|c|}
\hline \multirow{2}{*}{$\begin{array}{l}\text { Rice hybrid / } \\
\text { variety* }\end{array}$} & \multicolumn{2}{|c|}{ Yield (t/ha) } \\
\cline { 2 - 3 } & Grain & Biomass \\
\hline 28P09 & $2.68^{\mathrm{c}}$ & $8.27^{\mathrm{c}}$ \\
\hline DRRH3 & $2.36^{\mathrm{b}}$ & $7.32^{\mathrm{b}}$ \\
\hline KRH-4 $^{\mathrm{d}}$ & $2.91^{\mathrm{d}}$ & $9.02^{\mathrm{d}}$ \\
\hline WGL-14* & $2.32^{\mathrm{b}}$ & $7.19^{\mathrm{b}}$ \\
\hline CARI-6* & $2.09^{\mathrm{a}}$ & $6.48^{\mathrm{a}}$ \\
\hline CD $(\mathbf{P}=\mathbf{0 . 0 5})$ & 0.094 & 0.374 \\
\hline
\end{tabular}

Note: same superscript alphabets represent statistically similar values

Table.3 Nitrogen uptake and use efficiency of rice as influenced by cultivars

\begin{tabular}{|l|c|c|c|c|c|c|c|}
\hline \multirow{2}{*}{$\begin{array}{c}\text { Hybrid / } \\
\text { Variety* }\end{array}$} & \multicolumn{2}{|c|}{ N uptake (kg/ha) } & N Harvest & \multicolumn{4}{c|}{ Nitrogen use efficiency* } \\
\cline { 6 - 8 } & Grain & Biomass & index & AE & PE & AR & UE \\
\hline 28P09 & $31.91^{\mathrm{bc}}$ & $67.30^{\mathrm{c}}$ & $47.42^{\mathrm{a}}$ & $9.42^{\mathrm{c}}$ & $81.80^{\mathrm{d}}$ & $28.18^{\mathrm{d}}$ & $22.90^{\mathrm{c}}$ \\
\hline DRRH3 & $28.67^{\mathrm{b}}$ & $60.01^{\mathrm{b}}$ & $47.77^{\mathrm{a}}$ & $6.31^{\mathrm{b}}$ & $69.23^{\mathrm{c}}$ & $19.67^{\mathrm{c}}$ & $13.68^{\mathrm{b}}$ \\
\hline KRH-4 $^{\mathrm{b}}$ & $35.21^{\mathrm{c}}$ & $73.62^{\mathrm{d}}$ & $47.83^{\mathrm{a}}$ & $4.90^{\mathrm{ab}}$ & $48.34^{\mathrm{a}}$ & $15.55^{\mathrm{b}}$ & $7.48^{\mathrm{a}}$ \\
\hline WGL-14* $^{\mathrm{a}}$ & $27.69^{\mathrm{ab}}$ & $58.22^{\mathrm{b}}$ & $47.56^{\mathrm{a}}$ & $6.00^{\mathrm{b}}$ & $69.49^{\mathrm{c}}$ & $18.55^{\mathrm{c}}$ & $12.75^{\mathrm{b}}$ \\
\hline CARI-6* $^{\mathrm{b}}$ & $24.98^{\mathrm{a}}$ & $52.33^{\mathrm{a}}$ & $47.73^{\mathrm{a}}$ & $3.78^{\mathrm{a}}$ & $52.81^{\mathrm{b}}$ & $11.83^{\mathrm{a}}$ & $6.22^{\mathrm{a}}$ \\
\hline CD (P=0.05) & 3.443 & 5.129 & NS & & 4.01 & & \\
\hline
\end{tabular}

*AE: Agronomic efficiency (kg grain/ kg N applied): PE: Physiological efficiency (kg biomass/ $\mathrm{kg} \mathrm{N}$ uptake): AR: Apparent recovery (\%); UE: Utilization Efficiency ( $\mathrm{kg} / \mathrm{kg}$ )

Note: same superscript alphabets represent statistically similar values 
Table.4 Economics of rice cultivation and cultivars

\begin{tabular}{|l|c|c|r|r|}
\hline \multirow{2}{*}{$\begin{array}{l}\text { Rice hybrid / } \\
\text { variety* }\end{array}$} & \multicolumn{3}{|c|}{ Economics (Rs/ha) } & \multirow{2}{*}{$\begin{array}{c}\text { Benefit } \\
\text { Cost } \\
\text { Ratio }\end{array}$} \\
\cline { 1 - 4 } & $\begin{array}{c}\text { Cost of } \\
\text { cultivation }\end{array}$ & $\begin{array}{c}\text { Gross } \\
\text { returns }\end{array}$ & $\begin{array}{c}\text { Net } \\
\text { returns }\end{array}$ & \\
\hline 28P09 & 38601 & 48848 & $10247^{\mathrm{c}}$ & $1.26^{\mathrm{c}}$ \\
\hline DRRH3 & 38601 & 43271 & $4670^{\mathrm{a}}$ & $1.12^{\mathrm{a}}$ \\
\hline KRH-4 & 38601 & 53265 & $14664^{\mathrm{d}}$ & $1.38^{\mathrm{c}}$ \\
\hline WGL-14* & 35000 & 42524 & $7524^{\mathrm{b}}$ & $1.21^{\mathrm{b}}$ \\
\hline CARI Dhan-6* & 35000 & 38247 & $3247^{\mathrm{a}}$ & $1.09^{\mathrm{a}}$ \\
\hline CD (P=0.05) & - & - & 2008 & 0.072 \\
\hline
\end{tabular}

Note: same superscript alphabets represent statistically similar values

Table.5 Growth and yield attributes of rice as affected by nitrogen rates

\begin{tabular}{|c|c|c|c|c|c|c|}
\hline $\begin{array}{l}\text { Nitrogen rate } \\
\text { (kg/ha) }\end{array}$ & $\begin{array}{l}\text { Plant height } \\
\text { (cm) at } \\
\text { harvest }\end{array}$ & $\begin{array}{l}\text { Days to } 50 \% \\
\text { maturity }\end{array}$ & $\begin{array}{c}\text { Days to } \\
\text { maturity }\end{array}$ & $\begin{array}{c}\text { Panicles/ } \\
\mathbf{m}^{2}\end{array}$ & $\begin{array}{l}\text { Grains } \\
\text { /panicle }\end{array}$ & $\begin{array}{c}\text { Test } \\
\text { weight } \\
\text { (g) }\end{array}$ \\
\hline $\mathbf{0}$ & $96.9^{\mathrm{a}}$ & $94^{\mathrm{a}}$ & $121^{\mathrm{a}}$ & $225.8^{\mathrm{a}}$ & $99.7^{\mathrm{a}}$ & $20.28^{\mathrm{a}}$ \\
\hline 50 & $113.7^{b}$ & $99^{\mathrm{ab}}$ & $124^{\mathrm{ab}}$ & $272.0^{\mathrm{b}}$ & $108.3^{b}$ & $21.00^{\mathrm{a}}$ \\
\hline 100 & $124.3^{c}$ & $102^{b}$ & $127^{\mathrm{ab}}$ & $296.1^{c}$ & $113.2^{b}$ & $21.28^{\mathrm{a}}$ \\
\hline 150 & $127.2^{\mathrm{c}}$ & $105^{b}$ & $130^{\mathrm{b}}$ & $299.6^{c}$ & $114.8^{b}$ & $21.32^{\mathrm{a}}$ \\
\hline $\mathrm{CD}(\mathrm{P}=0.05)$ & 7.94 & 7.2 & 8.0 & 13.36 & 7.91 & NS \\
\hline $\begin{array}{l}\text { Interaction } \\
\text { (Cultivar } \mathbf{x} \mathbf{N})\end{array}$ & NS & NS & NS & NS & NS & NS \\
\hline
\end{tabular}

Note: same superscript alphabets represent statistically similar values; NS: Not significant

Table.6 Grain, biological yield, harvest index of rice as affected by nitrogen rates

\begin{tabular}{|l|c|c|c|}
\hline \multirow{2}{*}{$\begin{array}{l}\text { Nitrogen rate } \\
\text { (kg/ha) }\end{array}$} & \multicolumn{2}{|c|}{ Yield (t/ha) } & \multicolumn{1}{c|}{$\begin{array}{c}\text { Harvest } \\
\text { index }\end{array}$} \\
\cline { 2 - 4 } & Grain & Biological & \\
\hline $\mathbf{0}$ & $2.03^{\mathrm{a}}$ & $6.74^{\mathrm{a}}$ & $30.12^{\mathrm{a}}$ \\
\hline $\mathbf{5 0}$ & $2.33^{\mathrm{b}}$ & $7.39^{\mathrm{b}}$ & $31.53^{\mathrm{ab}}$ \\
\hline $\mathbf{1 0 0}$ & $2.71^{\mathrm{c}}$ & $8.01^{\mathrm{c}}$ & $33.90^{\mathrm{c}}$ \\
\hline $\mathbf{1 5 0}$ & $2.81^{\mathrm{c}}$ & $8.47^{\mathrm{c}}$ & $33.20^{\mathrm{bc}}$ \\
\hline $\mathbf{C D}(\mathbf{P}=\mathbf{0 . 0 5})$ & 0.145 & 0.579 & 2.02 \\
\hline
\end{tabular}

Note: same superscript alphabets represent statistically similar values 
Table.7 Nitrogen uptake and use efficiency of rice under varying nitrogen rates

\begin{tabular}{|c|c|c|c|c|c|c|c|}
\hline \multirow{2}{*}{$\begin{array}{l}\text { Nitrogen } \\
\text { rate (kg/ha) }\end{array}$} & \multicolumn{2}{|c|}{$\mathrm{N}$ uptake $(\mathrm{kg} / \mathrm{ha}$} & \multirow{2}{*}{$\begin{array}{l}\text { N Harvest } \\
\text { index }\end{array}$} & \multicolumn{4}{|c|}{ Nitrogen use efficiency* } \\
\hline & Grain & $\begin{array}{c}\text { Grain } \\
+ \\
\text { Straw }\end{array}$ & & $\underset{(\mathrm{kg} / \mathrm{kg})}{\mathrm{AE}}$ & $\begin{array}{c}\text { PE } \\
(\mathrm{kg} / \mathrm{kg})\end{array}$ & $\begin{array}{l}\text { AR } \\
(\%)\end{array}$ & $\begin{array}{c}\mathrm{UE} \\
(\mathrm{kg} / \mathrm{kg})\end{array}$ \\
\hline $\mathbf{0}$ & $21.86^{\mathrm{a}}$ & $49.02^{\mathrm{a}}$ & $44.59^{\mathrm{a}}$ & $\infty$ & $\infty$ & $\infty$ & $\infty$ \\
\hline 50 & $28.32^{b}$ & $59.75^{\mathrm{b}}$ & $47.41^{\mathrm{ab}}$ & $6.16^{b}$ & $57.55^{a}$ & $21.82^{\mathrm{a}}$ & $12.56^{\mathrm{ab}}$ \\
\hline 100 & $33.33^{b c}$ & $67.33^{b}$ & $49.50^{b}$ & $6.86^{c}$ & $65.80^{b}$ & $18.37^{b}$ & $12.09^{b}$ \\
\hline 150 & $35.25^{\mathrm{c}}$ & $73.08^{\mathrm{bc}}$ & $48.23^{b}$ & $5.23^{\mathrm{a}}$ & $69.55^{\mathrm{b}}$ & $16.08^{\mathrm{c}}$ & $11.20^{\mathrm{a}}$ \\
\hline $\mathrm{CD}(\mathbf{P}=\mathbf{0 . 0 5})$ & 5.367 & 7.922 & 3.942 & & 6.22 & & \\
\hline
\end{tabular}

*AE: Agronomic efficiency (kg grain/ kg N applied): PE: Physiological efficiency (kg biomass/ kg N uptake): AR: Apparent recovery (\%); UE: Utilization Efficiency (kg/kg)

Note: same superscript alphabets represent statistically similar values

Table.8 Economics of rice cultivation under varying nitrogen rates

\begin{tabular}{|c|c|c|c|c|}
\hline \multirow{2}{*}{$\begin{array}{l}\text { Nitrogen rate } \\
(\mathrm{kg} / \mathrm{ha})\end{array}$} & \multicolumn{3}{|c|}{ Economics (Rs/ha) } & \multirow{2}{*}{$\begin{array}{c}\text { Benefit } \\
\text { Cost } \\
\text { Ratio }\end{array}$} \\
\hline & $\begin{array}{c}\text { Cost of } \\
\text { cultivation }\end{array}$ & $\begin{array}{l}\text { Gross } \\
\text { returns }\end{array}$ & $\begin{array}{c}\text { Net } \\
\text { returns }\end{array}$ & \\
\hline $\mathbf{0}$ & 35745 & 38006 & $2241^{\mathrm{a}}$ & $1.06^{\mathrm{a}}$ \\
\hline 50 & 36589 & 43044 & $6535^{b}$ & $1.17^{b}$ \\
\hline 100 & 37433 & 48851 & $11418^{\mathrm{c}}$ & $1.30^{\mathrm{c}}$ \\
\hline 150 & 38277 & 50965 & $12688^{c}$ & $1.32^{\mathrm{c}}$ \\
\hline $\mathrm{CD}(\mathrm{P}=0.05)$ & - & - & 3128 & 0.113 \\
\hline
\end{tabular}

Note: same superscript alphabets represent statistically similar values

Table.9 Grain yield (t/ha) of rice as influenced by cultivar x nitrogen rate

\begin{tabular}{|c|c|c|c|c|}
\hline \multirow[t]{2}{*}{ Rice hybrid / variety* } & \multicolumn{4}{|c|}{ Nitrogen rate (kg/ha) } \\
\hline & $\mathbf{0}$ & 50 & 100 & 150 \\
\hline $28 P 09$ & $1.98^{\mathrm{aA}}$ & $2.45^{\mathrm{cB} 1}$ & $3.04^{\mathrm{cC}}$ & $3.22^{\mathrm{cC}}$ \\
\hline DRRH3 & $1.93^{\mathrm{aA}}$ & $2.32^{\mathrm{bcB} 1}$ & $2.57^{\mathrm{bC} 1}$ & $2.64^{\mathrm{bC} 1}$ \\
\hline KRH 4 & $2.55^{\mathrm{bAl}}$ & $2.79^{\mathrm{dB}}$ & $3.12^{\mathrm{cC}}$ & $3.18^{\mathrm{cC}}$ \\
\hline WGL 14* & $1.88^{\mathrm{aA}}$ & $2.16^{\mathrm{bB} 1}$ & $2.60^{\mathrm{bCl}}$ & $2.66^{\mathrm{bCl}}$ \\
\hline \multirow[t]{2}{*}{ CARI Dhan 6* } & $1.80^{\mathrm{aA}}$ & $1.96^{\mathrm{aA}}$ & $2.24^{\mathrm{aB} 1}$ & $2.36^{\mathrm{aB1}}$ \\
\hline & \multicolumn{2}{|c|}{ V at same $\mathbf{N}$} & \multicolumn{2}{|c|}{$\mathrm{V}$ at different $\mathrm{N}$} \\
\hline SEm \pm & \multicolumn{2}{|c|}{0.092} & \multicolumn{2}{|c|}{0.101} \\
\hline$C D(p=0.05)$ & \multicolumn{2}{|c|}{0.188} & \multicolumn{2}{|c|}{0.221} \\
\hline
\end{tabular}

${ }^{\text {abc }}$ denotes $\mathrm{V}$ at same $\mathrm{N} ;{ }^{\mathrm{ABC}}$ denotes $\mathrm{V}$ at different $\mathrm{N} ;{ }^{1}$ at par yields of $\mathrm{N}_{0}$ and $\mathrm{Nx}$ treatments.

Note: same superscript alphabets represent statistically similar values 
Table.10 Biomass yield ( $\mathrm{t} / \mathrm{ha}$ ) of rice as influenced by cultivar $\mathrm{x}$ nitrogen rate

\begin{tabular}{|l|c|c|c|c|}
\hline \multirow{2}{*}{$\begin{array}{l}\text { Rice hybrid / } \\
\text { variety* }\end{array}$} & \multicolumn{4}{|c|}{ Nitrogen rate (kg/ha) } \\
\hline $\mathbf{2 8 P 0 9}$ & $\mathbf{0}$ & $\mathbf{5 0}$ & $\mathbf{1 0 0}$ & $\mathbf{1 5 0}$ \\
\hline DRRH3 & $6.58^{\mathrm{b}}$ & $7.75^{\mathrm{c}}$ & $8.97^{\mathrm{c}}$ & $9.70^{\mathrm{c}}$ \\
\hline KRH 4 & $6.42^{\mathrm{ab}}$ & $7.34^{\mathrm{bc}}$ & $7.58^{\mathrm{b}}$ & $7.95^{\mathrm{b}}$ \\
\hline WGL 14* & $8.48^{\mathrm{c}}$ & $8.83^{\mathrm{d}}$ & $9.20^{\mathrm{c}}$ & $9.58^{\mathrm{c}}$ \\
\hline CARI Dhan $\mathbf{6}^{*}$ & $6.25^{\mathrm{ab}}$ & $6.83^{\mathrm{b}}$ & $7.67^{\mathrm{b}}$ & $8.01^{\mathrm{b}}$ \\
\hline & $5.99^{\mathrm{a}}$ & $6.20^{\mathrm{a}}$ & $6.61^{\mathrm{a}}$ & $7.11^{\mathrm{a}}$ \\
\hline SEm \pm & \multicolumn{3}{|c|}{ V at same N } & $\mathbf{V}$ at different $\mathbf{~ N}$ \\
\hline CD $(\mathbf{p}=\mathbf{0 . 0 5})$ & \multicolumn{2}{|c|}{0.284} & 0.309 \\
\hline
\end{tabular}

Note: Same superscript alphabets represent statistically similar values

Table.11 Net income (Rs/ha) of rice as influenced by cultivar $\mathrm{x}$ nitrogen rate

\begin{tabular}{|c|c|c|c|c|}
\hline \multirow{2}{*}{$\begin{array}{l}\text { Rice hybrid / } \\
\text { variety* }\end{array}$} & \multicolumn{4}{|c|}{ Nitrogen rate (kg/ha) } \\
\hline & $\mathbf{0}$ & 50 & 100 & 150 \\
\hline $28 P 09$ & $-99^{a}$ & $7099^{b}$ & $15646^{c}$ & $18343^{\mathrm{c}}$ \\
\hline DRRH3 & $-1035^{\mathrm{a}}$ & $4699^{\mathrm{ab}}$ & $7186^{\mathrm{a}}$ & $7831^{\mathrm{ab}}$ \\
\hline KRH 4 & $10578^{b}$ & $13376^{\mathrm{c}}$ & $17086^{\mathrm{c}}$ & $17618^{c}$ \\
\hline WGL 14* & $1628^{\mathrm{a}}$ & $5347^{\mathrm{ab}}$ & $11327^{b}$ & $11794^{b}$ \\
\hline \multirow[t]{2}{*}{ CARI Dhan 6* } & $130^{\mathrm{a}}$ & $1655^{\mathrm{a}}$ & $4847^{\mathrm{a}}$ & $6357^{\mathrm{a}}$ \\
\hline & \multicolumn{2}{|c|}{ V at same $\mathbf{N}$} & \multicolumn{2}{|c|}{$\mathrm{V}$ at different $\mathrm{N}$} \\
\hline SEm \pm & \multicolumn{2}{|c|}{1969} & \multicolumn{2}{|c|}{2157} \\
\hline $\mathrm{CD}(\mathrm{p}=\mathbf{0 . 0 5})$ & \multicolumn{2}{|c|}{4016} & \multicolumn{2}{|c|}{4721} \\
\hline
\end{tabular}

Table.12 Performance of hybrid and variety at recommended nitrogen (2016 season)

\begin{tabular}{|l|c|c|c|c|}
\hline \multirow{2}{*}{\multicolumn{1}{|c|}{$\begin{array}{c}\text { Hybrid / } \\
\text { Variety* }\end{array}$}} & \multicolumn{3}{c|}{ Grain Yield (t/ha) } & \multirow{2}{*}{ Mean } \\
\cline { 2 - 4 } & $\mathbf{2 0 1 5}$ & $\mathbf{2 0 1 6}^{*}$ & $\mathbf{2 0 1 8}^{* *}$ & \\
\hline KRH-4 & $3.12^{\mathrm{a}}$ & $5.24^{\mathrm{a}}$ & $4.15^{\mathrm{a}}$ & $4.17^{\mathrm{a}}$ \\
\hline WGL-14* & $2.60^{\mathrm{a}}$ & $4.31^{\mathrm{b}}$ & $3.76^{\mathrm{b}}$ & $3.56^{\mathrm{b}}$ \\
\hline CD $(\mathbf{P = 0 . 0 5})$ & 0.188 & 0.519 & 0.298 & 0.335 \\
\hline
\end{tabular}

$* \mathrm{CD}$ values based on 20 cultivars trial; ${ }^{*} \mathrm{CD}$ values based on 7 cultivars trial

Days to $50 \%$ flowering and maturity were significantly increased with $\mathrm{N}$ fertilization thus 100 and $150 \mathrm{~kg} \mathrm{~N}$ rate have significantly more values than no N. Nitrogen rates failed to alter 1000 grain weight of rice markedly. Interaction effects of variety and $\mathrm{N}$ rate on growth and yield attributes were nonsignificant. 


\section{Grain, biomass yield and harvest index}

Each successive increase of $50 \mathrm{~kg} \mathrm{~N}$ fertilizer from 0 to $100 \mathrm{~kg} / \mathrm{ha}$ has significantly pushed up the grain, biomass yield and harvest index of rice (Table 6). Nitrogen fertilization (mean of 50,100 and $150 \mathrm{~kg} \mathrm{~N}$ ) has improved the grain and biomass yield by 29.7 and $18.2 \%$ over no $\mathrm{N}$ control (2.03 and $6.74 \mathrm{t} / \mathrm{ha}$ ). Grain (biomass) yield increases have a quadratic improvement pattern i.e.14.8 (9.18), $16.31(8.68)$ and $3.69 \%(5.74 \%)$ increase with 50,100 and $150 \mathrm{~kg} \mathrm{~N}$ fertilization over immediate preceding rate. Harvest index improved with $100 \mathrm{~kg} \mathrm{~N}$ application over no N control only. Slight decline in harvest index was observed with increase in $\mathrm{N}$ dose from 100 to $150 \mathrm{~kg}$ and this decrease has made it to have at par values as $50 \mathrm{~kg} \mathrm{~N}$ rate. Grain yield exhibited linear response as determined by regression equation: $\mathrm{y}=0.272 \mathrm{x}+1.79$ ( $\mathrm{y}$ and $\mathrm{x}$ are yield in $\mathrm{t} / \mathrm{ha}$ and $\mathrm{N}$ rate in $\mathrm{kg} / \mathrm{ha}$ ) with a $\mathrm{R}^{2}$ value of 0.957 .

\section{Nitrogen uptake and use efficiency}

Nitrogen concentration in grain and straw were markedly altered by $\mathrm{N}$ fertilization (Figure 2). Application of $50 \mathrm{~kg}$ and $150 \mathrm{~kg} \mathrm{~N}$ has markedly improved $\mathrm{N}$ concentration of grain and straw over no $\mathrm{N}$ and $50 \mathrm{~kg} \mathrm{~N}$, respectively. Nitrogen uptake of grain, biomass and $\mathrm{N}$ harvest index varied with $\mathrm{N}$ rate (Table 7).

Gran (biomass) N uptake (kg/ha) was increased by 6.46 (11.09), 5.01 (7.33) and $1.92(5.75) \mathrm{kg}$ with $50,100,150 \mathrm{~kg} \mathrm{~N}$ application as compared to their immediate preceding rate. Nitrogen harvest index increased with $\mathrm{N}$ application and thus $100 \mathrm{~kg}$ $\mathrm{N}$ rate has markedly higher value than no $\mathrm{N}$ control. All $\mathrm{N}$ use efficiency indices increased with $\mathrm{N}$ fertilization. $\mathrm{AE}$ and $\mathrm{PE}$ values are highest with 100 and $150 \mathrm{~kg} \mathrm{~N} / \mathrm{ha}$, while AR and $\mathrm{UE}$ was highest at $50 \mathrm{~kg} \mathrm{~N}$ rate.

\section{Economics of nitrogen fertilization}

Nitrogen fertilization has significantly altered the economics (Rs/ha) of rice cultivation (Table 8). Cost of cultivation increased by Rs.944 for each $50 \mathrm{~kg} \mathrm{~N}$ fertilization. Net income and benefit cost ratio increased significantly with each successive increase of $50 \mathrm{~kg} \mathrm{~N}$ rate up to $100 \mathrm{~kg} / \mathrm{ha}$. Application of $150 \mathrm{~kg} \mathrm{~N}$ failed to enhance net income and BCR values significantly over $100 \mathrm{~kg} \mathrm{~N}$.

\section{Cultivar and nitrogen interaction for grain, biological yield and net income}

Grain, biomass yield and net income of rice differed significantly due to cultivar and $\mathrm{N}$ fertilization interaction (Table 9, Table 10 and Table 11). All cultivars have significant yield response to $\mathrm{N}$ fertilization up to $100 \mathrm{~kg}$ with the exception that biomass yield of '28P09' responded up to $150 \mathrm{~kg}$ rate. 'KRH-4' hybrid performance without $\mathrm{N}$ was as good as 'DRRH-3', 'WGL-14' and 'CARI Dhan-6' receiving $150 \mathrm{~kg} \mathrm{~N}$. Its superiority continued with $\mathrm{N}$ fertilization over other varieties with the exception that '28P09' has attained at par performance as KRH-4 at 100 and $150 \mathrm{~kg} \mathrm{~N}$ rate. Net income has brought out clear utility of $\mathrm{N}$ fertilization. Without $\mathrm{N}$ application, rice cultivation is economical with 'KRH-4' hybrid only and at $50 \mathrm{~kg} \mathrm{~N}$ also, it has no comparable treatment while 3 other cultivars have moved into profits (CARI Dhan-6 uneconomical). Application of $100 \mathrm{~kg} \mathrm{~N}$ has brought 28P09 hybrid on at par net income level as that of KRH-4. Potential performance of 28P09 was exhibited with $\mathrm{N}$ fertilization only and at $100 \mathrm{~kg} \mathrm{~N}$, gave its best performance. CARI Dhan-6 cultivation became economical with $100 \mathrm{~kg} \mathrm{~N}$ application only. WGL-14 variety has better economics than CARI Dhan-6 and DRRH-3 at 100 and $150 \mathrm{~kg} \mathrm{~N}$. Application of N beyond $100 \mathrm{~kg}$ was wasteful in all cultivars though grain yield response to $\mathrm{N}$ fertilization was linear. 


\section{Reassessment of hybrids performance over HYV}

Evaluation of best performing hybrid 'KRH-4' as compared to a high yielding variety WGL14 at recommended $\mathrm{N}$ dose $(100 \mathrm{~kg} / \mathrm{ha})$ during 2016 and 2018 (Table 12) and the data indicated consistent and significant yield improvement over WGL-14 variety. On an average at $100 \mathrm{~kg} \mathrm{~N}$ application KRH-4 has $17.14 \%$ yield advantage over the high yielding variety This data confirms that even at recommended dose of nitrogen, hybrids have exhibited their superiority.

Rice cultivars produced plants with a mean height of $115.5 \mathrm{~cm}$ that have 273.4 panicles/ $\mathrm{m}^{2}$, each panicle had 109 grains and 1000 grains weighed $20.97 \mathrm{~g}$ during the year 2015 . Rice hybrids 'KRH-4' and '28P09' have produced 9.5 and $8.1 \mathrm{~cm}$ taller plants than the mean value of $115.5 \mathrm{~cm}$. On the contrary, CARI Dhan-6 has $10.2 \mathrm{~cm}$ shorter plants than the mean value. Other two varieties have similar to mean plant height values. For panicles $/ / \mathrm{m}^{2}$, KRH-4 and WGL-14 have produced 44.1 and 33.1 more numbers than the mean (273.4) whereas CARI Dhan-6 has recorded $22 \%$ number of panicles than the mean. Grains/ panicle of 28P09 hybrid was $13.6 \%$ higher number of grains than the mean value (109) and DRRH-3 hybrid has 7.9\% lesser values than the mean. Huge variation was seen for 1000 grain weight (16.63 $25.07 \mathrm{~g}$ ) among cultivars. WGL-14 and CARI Dhan-6 have $-20.7 \%$ and $19.6 \%$ deviations from the mean value $(20.97 \mathrm{~g})$.

Rice crop has a mean grain, biological yield $(\mathrm{t} / \mathrm{ha})$ and harvest index values of 2.477 .65 and 32.3 , respectively. Taller plants $(9.5 \mathrm{~cm})$ of KRH-4 hybrid coupled with $16.13 \%$ higher number of panicles $/ \mathrm{m}^{2}$ (with grains/ panicle and test weight values at par with mean values) together has brought in $17.9 \%$ higher (biomass and grain) yields over the mean. rice hybrid '28P09' too on account of $8.1 \mathrm{~cm}$ taller plants and $13.6 \%$ higher grains/panicle than the mean values (with panicles $/ \mathrm{m}^{2}$ and 1000 grain weight remained at par with mean values) have exhibited 8.5 and $8 \%$ grain and biomass yield improvements. Rice variety 'WGL-14' despite of producing $11.1 \%$ higher number of panicle $/ \mathrm{m}^{2}$, owing to $20.7 \%$ lower 1000 grain weight than the mean $(20.97 \mathrm{~g})$ has recorded significantly lower yields $(6.1 \%)$ than the mean yield. Similarly, the lower panicle production of DRRH-3 (5.4\%) got compensated by $18.7 \%$ higher seed weight than the mean has brought its performance at par with WGL-14. Local improved variety CARI Dhan-6 remained the poor performer due to $10.2 \mathrm{~cm}$ shorter plants and $25.7 \%$ lesser panicle $/ \mathrm{m}^{2}$ production than the mean. Its heavier grains $(19.6 \%$ higher than mean) failed to compensate the lesser panicle $/ \mathrm{m}^{2}$ and thus had $15.4 \%$ lower yield than the mean and thus was a poor performer. In general, rice crop took 25 day time from $50 \%$ flowering stage to reach physiological maturity. As cultivars tested belonged to same medium group of maturity (120-130 days), the differences remained non significant. Superior performance of hybrids and varieties over traditional and local improved variety of the current study was corroborated by the findings of Singh et al., (2009) with hybrids and Sharma et al., (2016) with HYV.

The current experimental research farm site was located in a place surrounded by forest trees and the small birds (sparrows) are eating the grains. As per Forest law, they can't be controlled as comes under protected category. Current yields reported are such uniformly bird damaged study. In general, the yields are $25 \%$ lower than the actual and yields. In farmers' fields, where paddy is grown in continuous stretches, yield losses due to birds was quite lower and in significant as spread over many fields. Cultivars varied for their $\mathrm{N}$ use efficiency indicators vastly and are highest with 28P09. 
Current experimental site clay loam soil was low for available nitrogen $(258 \mathrm{~kg} / \mathrm{ha})$ and was subjected to heavy rains in August and September months coinciding with $\mathrm{N}$ top dressings that might have washed the mineralised $\mathrm{N}$ out of the field. Nitrogen response of rice to $\mathrm{N}$ fertiliser was assessed with uniform recommended $\mathrm{P}_{2} \mathrm{O}_{5}$ and $\mathrm{K}_{2} \mathrm{O}$ fertilizers $(60 \mathrm{~kg}$ each) and micronutrient deficiency and response (iron, zinc etc.) was not reported. Thus $\mathrm{N}$ responses were clearly captured. Nitrogen nutrition of rice has enhanced the plant height, panicles $/ \mathrm{m}^{2}$ and grains/panicle production greatly. Plant height was increased by $16.8,10.6$ and $2.9 \mathrm{~cm}$ with addition of 50,100 and $150 \mathrm{~kg} \mathrm{~N}$ fertilizer over their immediate preceding rate. The figures for panicles $/ \mathrm{m}^{2}$ were 46.2, 24.1 and 7.5 and for grains/panicle were 8.6, 4.9 and 1.6. Test weight did not vary much with $\mathrm{N}$ dose. On account of 5.8, 2.8 and 11\% higher panicles $/ \mathrm{m}^{2}$, grains/panicle and 1000 grain weight, $\mathrm{N}$ fertilization has increased grain yields by $25.2 \%$ over no $\mathrm{N}$ control (2.09 t/ha). This increase was 15.3, 16.3 and 3.7 with 50, 100 and $150 \mathrm{~kg} \mathrm{~N}$ application over 0,50 and $100 \mathrm{~kg} \mathrm{~N}$, respectively. On account of above increases in yield attributes along with $5.4 \%$ taller plants in $\mathrm{N}$ fertilized crop has brought in $17.8 \%$ increases in biomass yield than no $\mathrm{N}$ control (6.75 t/ha). Per cent increase in grain yield was far ahead of biomass yield up to 100 $\mathrm{kg} \mathrm{N}$ and at $150 \mathrm{~kg} \mathrm{~N}$, biomass yield surpassed grain yield increases indicating luxury consumption of $\mathrm{N}$. Nitrogen fertilization has increased the time taken for $50 \%$ flowering by 8 days as compared no $\mathrm{N}$ control (94 days). Maturity time is increased by 6 days. The above increases in yields (straw and grain) have translated into net income and $100 \mathrm{~kg} \mathrm{~N}$ is ideal. Mean nitrogen harvest index of the current study are (44.59) is quiet lower than the reported values of $64 \%$ by Fageria and Baligar (2001). This was ascribed to loss of $25 \%$ grain yield by bird damage. Nitrogen harvest index $(\mathrm{NHI})$ of rice increased in $\mathrm{N}$ fertilized crop by 3.71 as compared to no $\mathrm{N}$ control (44.64). This indicates that for better yields, a NHI of 49.5 is required that is attained with $100 \mathrm{~kg} \mathrm{~N}$ fertilization at the study region. $\mathrm{N}$ uptake of rice increased on account of increased straw / grain yield and their $\mathrm{N}$ concentrations with $\mathrm{N}$ dose (Figure 2). Agronomic efficiency (AE) of $\mathrm{N}$ increased with $\mathrm{N}$ fertilization up to $100 \mathrm{~kg}$ and thereafter declined. The grain yield increase was 0.30 , 0.38 and $0.11 \mathrm{t} / \mathrm{ha}$ for $1^{\text {st }}, 2^{\text {nd }}$ and $3^{\text {rd }} 50 \mathrm{~kg} \mathrm{~N}$ addition. The decreasing rate of yield increases with constant rate of $\mathrm{N}$ increase (50 $\mathrm{kg}$ ) has resulted in decrease in $\mathrm{AE}$. Physiological Efficiency (PE) dependent on ratio of biomass increases to $\mathrm{N}$ uptake increases in fertilized plot over no $\mathrm{N}$ application.

Both biomass and $\mathrm{N}$ uptake increased with $\mathrm{N}$ fertilization over control and thus have highest values at $150 \mathrm{~kg} \mathrm{~N}$ application. Apparent recovery (AR) was highest with $50 \mathrm{~kg} \mathrm{~N}$ $(22.18 \%)$ and decreased with increased $\mathrm{N}$ rate to the lowest of 16.12 with $150 \mathrm{~kg} \mathrm{~N}$ on account of increasing numerator ( $\mathrm{N}$ dose). Utilization Efficiency (UE), a product of AR and PE followed their trend.

Better performance of KRH-4 hybrid even with no $\mathrm{N}$ fertilization of current study may be ascribed to their larger, deeper, and more vigorous root systems (Yang and Sun 1986; Zhang et al., 2009) generated with $\mathrm{P}$ and $\mathrm{K}$ fertilizers in current study. The large volume of roots might have enabled hybrid to draws more soil $\mathrm{N}$ (Hunag et al., 2017) while fertilizer $\mathrm{N}$ contributions remained similar for a hybrid and high yielding variety. Superior performance of hybrid (KRH-4) at $100 \mathrm{~kg} \mathrm{~N}$ dose over WGL-14 variety for 3 seasons (2015, 2016 and 2018) has confirmed that hybrids have efficient nutrient absorption and use mechanisms for higher performance at both low and optimum dose of nitrogen. This was proved by studies of Hunag et al., (2017). 
From the above study it is concluded that scope lies for replacing local variety 'CARI Dhan-6' by new high yielding cultivars (KRH4 and 28P09) with a yield gain of 28.2-39.2\%.

There is linear response to $\mathrm{N}$ fertilization up to $150 \mathrm{~kg} / \mathrm{ha}$ at the site, but $100 \mathrm{~kg} \mathrm{~N}$ was economically optimum. Cultivar and nitrogen interaction reveal that scope lies for attaining current yields with low or no $\mathrm{N}$ fertilization by choosing $\mathrm{KRH}_{4}$ hybrid. KRH-4 and 28P09 are promising with low and recommended dose of $\mathrm{N}$, respectively. Above hybrids have exhibited yield superiority over varieties even at optimum $\mathrm{N}$ dose $(100 \mathrm{~kg})$ in experimental region.

\section{References}

Amanullah, M., Natarajan, S., Vanathi, D., Ramasamy, S. and Sathyamoorthi, K. 2007. Lowland rice in coastal saline soils - a review. Agricultural Science Digest. 28 (4): 235-238.

Courtois, B., Bartholome, D. Chaudhary, G. McLaren, C.H. Misra, N.P. Mandal, S. Pandey, T. Paris, C. Piggin, K. Prasad, A.T. Roy, R.K. Sahu, V.N. Sahu, S. Sarkarung, S.K. Sharma, A. Singh, H.N. Singh, O.N. Singh, N.K. Singh, R.K. Singh, R.K. Singh, S. Singh, P.K. Sinha, B.V.S. Sisodia and R. Takhur. 2001. Comparing farmers and breeders rankings in varietal selection for low-input environments: A case study of rainfed rice in eastern India. Euphytica. 122: 537-550.

Damodaran, V., Saren, B. K. ; Ravisankar, N. ; Bommayasamy, N. 2012. Influence of time of planting, spacing, seedling number and nitrogen management practices on productivity, profitability and energetics of rice (Oryza sativa) in island ecosystem. Madras Agricultural Journal. 99 (7/9): 538544.

DOES (Directorate of Economics and Statistics). 2018. Agriculture: Major Crops in A\&N Islands. DOES, Andaman and Nicobar Administration, Port Blair.
DRD (Directorate of Rice Development). 2018. Hybrid Varieties of Rice in India. Directorate of Rice Development, Patna. drdpat.bih.nic.in/ Hybrid-RiceVarieties.htm.

Fageria, N. K. and Baligar, V. C. 2001. Lowland rice response to nitrogen fertilization. Communications in Soil Science and Plant Analysis. 32 (9): 1405-1429.

FAO (Food and Agriculture Organization of the United Nations). 2004. Hybrid rice for food security. http://www.fao. org/rice2004/en/fsheet/factsheet6.pdf

Gangaiah, B., Subramani, T., Swain. S., Velmurugan, A., Nanada, B.K., Damodaran, V., Lohit Kumar, K and Hundu, M.S. 2016. Vulnerability assessment and adaptation led mitigation strategies of Andaman and Nicobar islands farming to claimte change. Pp. 54-55. In: Annual Report 2015-16., ICAR- Central Island Agricultural Research Institute, Port Blair, Andaman and Nicobar islands.

Ganesh Kumar, B., R. Sendhil, P. Venkatesh, R. Raja, V. Jayakumar and S. Jeyakumar. 2009. Socio-economic Impact Assessment of Livelihood Security in Agriculture, Animal Husbandry and Aquaculture on the Tsunami-hit Lands of Andaman. Agricultural Economics Research Review. 22: 483-494.

Gomez, K. A. and Gomez, A. A. 1984. Statistical Procedures for Agricultural Research. John Wiley and Sons, Inc. London, UK, $\left(2^{\text {nd }} E d\right)$.

Huang, M, Peng Jiang, Shuanglü Shan,Wei Gao, Guohui Ma,Yingbin Zou, Norman Uphoff and Longping Yuan. 2017. Higher yields of hybrid rice do not depend on nitrogen fertilization under moderate to high soil fertility conditions. Rice (N Y): 10: 43.

Jackson, M.L. 1973. Soil Chemical Analysis, Prentice Hall Publication, New Delhi, p.184.

Mandal, A.B., Elanchezhian, R. and Majaumdar, N.D. 2004. Genetic management for increased productivity of rice in Andaman and Nicobar Islands. ICAR- Central Agricultural Research Institute, Port Blair, Andaman and Nicobar Islands, $196 \mathrm{p}$.

Olsen, C.R., Cole, C.V., Wantanabe, F.S. and Dean, L.A. 1954.Estimationofavailable phosphorus in soils by extraction with 
sodium bicarbonate. USDA Circ. No. 939 Washington. pp. 19.

Shanmugasundaram, B. and Ponnusamy, K.A. 2009. Effect of tsunami on the socioeconomic condition of rice farmers.Indian Res. J Ext. Edu. 9 (2): 120-124.

Sharma, S. K., Singh, Y.V., Suruchi Tyagi and Arti Bhatia. 2016. Influence of rice varieties, nitrogen management and planting methods on methane emission and water productivity. Paddy Water Environment, 14:325-333.

Singh, A.K., Birendra Kumar, Baghel, R.S. and Singh, R.B. 2009 Sustainability of Hybrid Rice Technology vis a vis Inbred Rice in Uttar Pradesh. Indian Research Journal of Extensuon Education. 9 (2): 22-25.

Singh Dhyan, Chhinkar, P.K. and Dwivedi, B.S. 2005. Manual on Soil, Plant and Water Analysis. Westville Publishing House, New Delhi.

Singh, P.K., Gautam, R.K., Zamir Ahmed, S.K., Singh, K. Awnindra, Sakthivel, K and Dam Roy S., 2014. Farmers' participatory seed production and adoption of rice varieties in Andaman and Nicobar Islands: a success story, Bulletin, CIARI, Port Blair pp.1-32.

Singh, N.T., Mongia, A.D. and Ganeshmurthy, A.N. 1988. Soils of Andaman and Nicobar Islands. CARI Technical Bulletin1. 1-64 pp. Central Agricultural Research Institute, Port
Blair, Andaman \& Nicobar Islands.

Subbiah, B.V. and Asija, G.L. 1956. A rapid procedure for estimation of available nitrogen in soils. Current Science. 5: 656659.

Subramani, T., Raje, R., Ambast, S.K, Ravishankar, N., Zamir Ahmed, S.K., Damodaran, V. and Bommayasamy. N. 2014. Evaluation of long duration rice varieties for enhancing productivity and profitability under Island ecosystem. Journal of the Andaman Science Association 19(1): 14-18.

Velmurugan, A., Swarnam, T.P. and Rattan Lal. 2015. Effect of land shaping on soil properties and crop yield in tsunami inundated coastal soils of Southern Andaman Island. Agriculture, Ecosystems \& Environment. 206: 1-9.

Yang, X. and Sun, X. 1986. Physiological characteristics of hybrid rice roots. In: Hybrid Rice. International Rice Research Institute, Los Baños, p 281.

Zhang, H., Xue, Y., Wang, Z, Yang, J. and Zhang, J. 2009. Morphological and physiological traits of roots and their relationships with shoot growth in "super" rice. Field Crops Research. 13:31-40.

\section{How to cite this article:}

Gangaiah, B., Adamala Sirisha, S. Swain and Subramani, T. 2019. Differential Response of Rice Hybrids and Varieties to Nitrogen Fertilization and their Exploitation in Andaman \& Nicobar Islands. Int.J.Curr.Microbiol.App.Sci. 8(09): 1382-1396.

doi: https://doi.org/10.20546/ijcmas.2019.809.159 